\title{
AVALIAÇÃO DA FUNÇÃO PULMONAR ASSOCIADA AO EXERCíCIO AERÓBICO EM PACIENTES QUE REALIZAM HEMODIÁLISE
}

\author{
Barbara dos Santos Fahur ${ }^{1}$, Lin Sheng Yen ${ }^{1}$, Giovana Navarro Bertolini Ferrari ${ }^{2}$, Susimary Aparecida \\ Trevizan Padulla ${ }^{3}$, Regina Coeli Vasques de Miranda ${ }^{3}$
}

\begin{abstract}
${ }^{1}$ Discente do Curso de Graduação em Fisioterapia, ${ }^{2}$ Discente do Curso de Pós-Graduação em Fisioterapia, ${ }^{3}$ Docente do Departamento de Fisioterapia da FCT/UNESP, Presidente Prudente, SP. barbarafahur@gmail.com
\end{abstract}

Agência Financiadora: FAPESP

\section{RESUMO}

A natureza progressiva da doença renal crônica (DRC) gera um desequilíbrio e disfunção a longo prazo. A presença de distúrbios da mecânica respiratória, limitação do fluxo aéreo distal e redução da capacidade de difusão pulmonar é freqüente nessa população. O objetivo foi avaliar os efeitos de um programa de exercício durante oito semanas sobre a Capacidade Vital (CVF), Volume Expiratório Forçado no Primeiro Segundo (VEF1) e Índice de Tiffeneau (VEF1/CVF). Participaram da pesquisa 20 indivíduos. A função pulmonar foi avaliada através da espirometria. Para análise estatística foi utilizado o Teste de KurskalWallis. Para localizar a diferenças entre as variáveis foi aplicado o Teste Post Hoc de Bonferroni. A espirometria mostrou redução significativa dos valores espirométricos (CVF e VEF1) em relação aos valores preditos $(p=0,0001)$. Não houve mudança significante entre esses valores pré e pós-exercício. $O$ índice de Tiffeneau não mostrou diferença estatística em nenhum momento. Concluímos que programas de exercício aeróbico com pacientes em hemodiálise não produzem efeito significativo sobre a função pulmonar.

Palavras-chave: doença renal crônica, função pulmonar, exercício.

\section{EVALUATION OF PULMONARY FUNCTION IN AEROBIC EXERCISE ASSOCIATED PATIENTS ON HEMODIALYSIS}

\section{ABSTRACT}

The progressive nature of kidney disease (CKD) creates an imbalance and dysfunction in the long term. The presence of disorders of respiratory mechanics, airflow limitation and reduction of distal pulmonary diffusion capacity is frequent in this population. The aim was to evaluate the effects of an exercise program for eight weeks on the Vital Capacity (FVC), forced expiratory volume in one second (FEV1) and Tiffeneau index (FEV1/FVC). Subjects were 20 individuals. Lung function was assessed by spirometry. Statistical analysis was used to test Kurskal-Wallis. To find the difference between variables was applied Bonferroni Post Hoc Test. Spirometry showed a significant reduction in spirometric values (FVC and FEV1) compared to predicted values $(p=0.0001)$. There was no significant change between these values before and after exercise. The Tiffeneau index showed no statistical difference in no time. It was concluded that aerobic exercise programs with hemodialysis patients do not produce significant effect on lung function.

Keywords: chronic kidney disease, pulmonary function, exercise. 


\section{INTRODUÇÃO}

A doença renal crônica (DRC) é caracterizada por lesão renal e perda irreversível e progressiva da função dos rins, os quais em fases mais avançadas não mantêm a homeostase do indivíduo, necessitando este de terapia de substituição (ROMÃO JUNIOR, 2004).

O processo de hemodiálise é realizado,em média, por quatro horas e três vezes por semana (MAGALHÃES et al., 2004). O tratamento da DRC por meio de diálise melhora o tempo de sobrevida dos pacientes com doença renal, entretanto, deve-se ficar atento às complicações que implicam na qualidade de vida (CASTRO et al., 2004). Verifique na referência consta data de 2003.

A natureza progressiva da DRC gera um desequilíbrio ou disfunção a longo prazo. A utilização exacerbada de medicamentos e a dependência de uma máquina contribuem para debilitar o doente renal e comprometer sua qualidade de vida (RIELLA, 2003; SANTOS, 2006).

As alterações da função respiratória são freqüentes em pacientes com DRC em tratamento hemodialítico, caracterizadas pela fraqueza muscular, distúrbios da mecânica respiratória, limitação do fluxo aéreo distal e redução da capacidade de difusão pulmonar (COELHO et al., 2006; SHARDONG et al., 2008). Estudo sobre avaliações pulmonares realizadas por Schardong et.al.,mostraram valores de capacidade vital forçada (CVF), volume expiratório forçado no primeiro segundo (VEF1) e o índice Tiffeneau (VEF1/CVF), muito abaixo do previsto em 30 pacientes portadores de DRC submetidos à hemodiálise (SHARDONG et al., 2008).

A compreensão das limitações encontradas nesta população parece ser relevante na realização desta pesquisa, no sentido de contribuir para futuros programas padronizados de exercícios físicos com o objetivo de melhorar a função pulmonar nestes pacientes.

\section{OBJETIVO}

Avaliar os efeitos de um programa de atividade física durante oito semanas sobre a função pulmonar de pacientes com DRC que realizam hemodiálise.

\section{METODOLOGIA}

Foram selecionados 27pacientes (18 homens e 9 mulheres) com idade entre 35 - 75 anos, com o diagnóstico de DRC em programa de hemodiálise no Instituto do Rim da Santa Casa de Misericórdia de Presidente Prudente SP.

Foram excluídos da pesquisa os pacientes com doenças neurológicas e pacientes impossibilitados a realizar o trabalho de estudo tais como: amputação e trombose venosa.

A prova de função pulmonar foi feita segundo os critérios do I Consenso Brasileiro sobre Espirometria (SOCIEDADE BRASILEIRA DE PNEUMOLOGIA E TISIOLOGIA, 1996) e as Diretrizes para Testes de Função Pulmonar (SOCIEDADE BRASILEIRA DE PNEUMOLOGIA, 2002), com a utilização do espirômetro a volume da marca COSMED $\AA$ tipo "Pony Fx" (Registro Anvisa no 8018193001). A espirometria avaliou a Capacidade Vital Forçada (CVF), o Volume Expiratório Forçado no primeiro minuto (VEF1) e o índice de Tiffeneu (VEF1/CVF).

Todas as avaliações foram realizadas antes e após o programa de exercício, o qual foi realizado com cicloexercitador portátil com carga regulável, três vezes por semana durante 
oito semanas, com duração de 20 minutos cada sessão.

Para análise estatística, foi aplicado o teste de Anova One Way, porém quando o pressuposto da homogeneidade das variâncias não foi atendido procedeu-se 0 Teste de Kurskal-Wallis. Para localizar a diferenças entre as variáveis foi aplicado o Teste Post Hoc de Bonferroni.

\section{RESULTADOS}

Após análise estatística dos resultados, foi observado que o valor predito de CVF demonstrou-se superior do ponto de vista estatístico aos valores de CVF pré e pós intervenção, porém não foi verificado diferenças significativas sobre CVF entre os momentos pré e pós intervenção (Tabelas 1 e 2).

Tabela 1. Capacidade Vital Forçada (CVF). Valores predito e pré-treino.

\begin{tabular}{llll}
\hline & N & $\begin{array}{l}\text { Média } \\
\text { (L/seg) }\end{array}$ & DP \\
\hline Predito & 27 & 3,73 & 0,80 \\
Pré-treino & 27 & 2,66 & 0,93 \\
\hline
\end{tabular}

$p=0,0001$

Tabela 2. Valores de CVF pré e pós-treino.

\begin{tabular}{llll}
\hline & $\mathbf{N}$ & $\begin{array}{l}\text { Média } \\
\text { (L/seg) }\end{array}$ & DP \\
\hline Pré-treino & 27 & 2,66 & 0,93 \\
Pós-treino & 27 & 2,81 & 1,02 \\
\hline
\end{tabular}

NS

O mesmo se observa para a variável

VEF1, onde o valor predito se apresenta superior aos momentos pré e pós intervenção, mas sem influência significativa sobre esta variável pré a pós intervenção (Tabelas 3 e 4).
Tabela 3. Volume Expiratório Forçado no Primeiro Minuto (VEF1). Valores predito e prétreino.

\begin{tabular}{llll}
\hline & N & $\begin{array}{l}\text { Média } \\
\text { (L/seg) }\end{array}$ & DP \\
\hline Predito & 27 & 2,99 & 0,64 \\
Pós-treino & 27 & 2,15 & 0,78 \\
\hline
\end{tabular}

$p=0,0001$

Tabela 4. Valores VEF1 pré e pós-treino.

\begin{tabular}{llll}
\hline & $\mathbf{N}$ & $\begin{array}{l}\text { Média } \\
(\mathrm{L} / \mathbf{s e g})\end{array}$ & DP \\
\hline Pré treino & 27 & 2,15 & 0,78 \\
Pós treino & 27 & 2,31 & 0,83 \\
\hline NS & & &
\end{tabular}

Já na variável índice de Tiffeneau não foram encontradas diferenças significativas entre os momentos, além de que os pacientes não apresentaram valores de VEF1/CVF acima de $90 \%$ do previsto, valor considerado como ponto de corte para a normalidade de adultos brasileiros $^{9}$ (Tabela 5).

Tabela 5. Índice de Tiffeneau. Valores preditos, pré e pós-treino.

\begin{tabular}{llll}
\hline & N & Média & DP \\
\hline Predito & 27 & 78,78 & 3,15 \\
Pré-treino & 27 & 80,75 & 7,35 \\
Pós-treino & 27 & 82,33 & 8,90 \\
\hline
\end{tabular}

NS

Os resultados sugerem que na amostra estudada a intervenção não levou a modificações significativas sobre as variáveis CVF, VEF1 e Tiff, e que na DRC os valores de CVF e VEF1 encontrados são inferiores que o previsto, e possivelmente estas variáveis são mais influenciadas por esta patologia.

\section{DISCUSSÃO}

A baixa capacidade funcional para atividades diárias é resultado de um conjunto de fatores como alterações cardiopulmonares, 
musculoesqueléticas,

neurológicas,

hidroeletrolíticas e endocrinometabólicas, induzindo à dispnéia, síndrome urêmica, fadiga, dor em membros inferiores, hipertensão arterial sistêmica, anemia e fraqueza muscular generalizada. As alterações respiratórias, que ocorrem devido à doença e ao seu próprio tratamento, estão relacionadas, sobretudo, à hipotrofia muscular de, à alteração do transporte, extração e consumo de oxigênio, à deficiência de vitamina $D$ etc. Estudos com indivíduos portadores de DRC demonstram que a força muscular respiratória e as variáveis de função pulmonar estão abaixo dos valores de normalidade (JATOBÁ et al., 2008).

Mesmo o presente estudo apresentando perfil de amostra distinta, sendo composta de 27 indivíduos adultos jovens e idosos, foi possível observar que todos esses pacientes apresentaram redução significativa dos valores espirométricos (CVF e VEF1) em relação ao valor predito, o que corrobora com os dados descritos pela literatura atual, observados nessa população (SHARDONG et al., 2008; JATOBÁ et al., 2008; CURY et al., 2010; BIANCHI et al., 2009; KOVELIS et al., 2008).

É de conhecimento da população que o exercício físico produz efeitos benéficos. Estudos mostram resultados positivos associando atividade física ao paciente que realiza hemodiálise (COELHO, 2006), mesmo que a DRC seja um fator limitante do condicionamento físico ideal (JOHANSEN, 2007). Apesar dessa afirmação, esse estudo não mostrou alteração significativa sobre a função pulmonar desses indivíduos, assim como também não houveram reduções das variáveis analisadas após o programa de exercício, sugerindo uma manutenção no quadro pulmonar desses pacientes.
Embora o exercício físico não produzira efeitos significativos sobre a função pulmonar dos indivíduos, estudos demonstram que o exercício aeróbico prolongado durante a hemodiálise produz efeitos benéficos em relação ao aumento da remoção de solutos devido à vasodilação em musculatura esquelética, propiciando maior remoção de catabólitos (KONG et al., 1999), alterações musculares morfológicas, mostrando aumento de área de seccção tranversa e redução de atrofia de fibras musculares, assim como, aumento na capilarização muscular (SAKKAS et al., 2003); melhora de força e performance física (STORER et al., 2005) e diminuição do uso de medicamento anti hipertensivo (MILLER et al., 2002; PARSONS et al., 2004).

\section{CONCLUSÃO}

Os resultados desta pesquisa mostraram que o exercício aeróbico não produz efeitos significativos sobre a função pulmonar.

\section{REFERÊNCIAS}

Bianchi PDA, Barreto SSM, Thomé FS, Klein AB. Repercussão da Hemodiálise na Função Pulmonar de Pacientes com Doença Renal Crônica Terminal. J Bras Nefrol 2009;31(1):2531.

Castro M, Caiuby A, Draibe S, Canziani EM. Qualidade de vida em pacientes com insuficiência renal crônica em hemodiálise avaliada através do instrumento genérico SF-36. Rev de Assoc Med Bras 2003; 49:245-9. http://dx.doi.org/10.1590/S0104-

\section{$\underline{42302003000300025}$}

Coelho DM, Castro AM, Tavares HA, Abreu PCB, Glória RR, Duarte $\mathrm{MH}$ et al. Efeitos de um programa de exercícios físicos no 
condicionamento de pacientes em hemodiálise. Jornal Brasileiro Nefrologia 2006;28:121-7.

Cury JL, Brunetto AF, Aydos RD. Efeitos negativos da IRC na função pulmonar e a capacidade funcional. Rev Bras Fisioter. 2010;14(2):91-8. http://dx.doi.org/10.1590/S1413$\underline{35552010005000008}$

Jatobá JP, Amaro WF, Andrade AP, Cardoso FP, Monteiro AM, Oliveira MA. Avaliação da função pulmonar, força muscular respiratória e teste de caminhada de seis minutos em pacientes portadores de doença renal crônica em hemodiálise. J Bras Nefrol 2008;30(4):280-7.

Johansen KL. Exercise in the end-stage renal disease population. Journal of the American Society of Nephrology. 2007;18:1845-54. http://dx.doi.org/10.1681/ASN.2007010009

Kong $\mathrm{CH}$, Tattersall JE, Greenwood RN, Farringyon G. The effect of exercise during haemodialysis on solute removal. Nephrol Dial Transplant. 1999;14(12):2927-31. http://dx.doi.org/10.1093/ndt/14.12.2927

Kovelis D, Pitta F, Probst VS, Peres CPA, Delfino VDA, Mocelin AJ, et.al. Função pulmonar e força muscular respiratória em pacientes com doença renal crônica submetidos à hemodiálise. J Bras Pneumol. 2008;34(11):907-912.

Magalhães HG, Pinto TA, Reboredo MM, Fonseca FD, Almeida PC Análise da eficiência do tratamento fisioterapêutico em pacientes com insuficiência renal crônica em hemodiálise. Anais do 2o Congresso Brasileiro de Extensão Universitária. Belo Horizonte, 2004.

Miller BW, Cress CL, Johnson ME, Nichols DH, Schnitzler MA. Exercise during hemodialysis decreases the use of antihypertensive medications. Am J Kidney Dis. 2002;39(4):828-33. http://dx.doi.org/10.1053/ajkd.2002.32004
Parsons TL, Toffelmire EB, King-VanVlack CE. The effect of an exercise program during hemodialysis on dialysis efficacy, blood pressure and quality of life in end-stage renal disease (ESRD) patients. Clin Nephrol. 2004;61(4):26174.

Riella MC. Princípios de nefrologia e distúrbios hidroeletrolíticos. Rio de Janeiro: Guanabara Koogan, 2003.p.649-60.

Romão Junior JE. Doença renal crônica: definição,epidemiologia e classificação. J Bras Nefrol. 2004;26:1-3.

Sakkas GK, Sargeant AJ, Mercer TH, Ball D, Koufaki P, Karatzaferi C, et al. Changes in muscle morphology in dialysis patients after 6 months of aerobic exercise training. Nephrol Dial Transplant. 2003;18(9):1854-61.

http://dx.doi.org/10.1093/ndt/gfg237

Santos PR. Relação do sexo e da idade com nível de qualidade de vida em renais crônicos hemodialisados. Revista Associação Médica Brasileira. 2006;52:356-9.

http://dx.doi.org/10.1590/S0104$\underline{42302006000500026}$

Shardong TJ, Lukrafka JL, Garcia VD. Avaliação da função pulmonar e da qualidade de vida em pacientes com doença renal crônica submetidos à hemodiálise. Jornal Brasileiro Nefrologia, 2008;30(1):40-7.

Sociedade Brasileira de Pneumologia. Diretrizes para Testes de Função Pulmonar. J Pneumol 2002;28(3):1-238.

Sociedade Brasileira de Pneumologia e Tisiologia. I Consenso Brasileiro sobre Espirometria. J Pneumol 1996; 22(3):105-64.

Storer TW, Casaburi R, Sawelson S, Kopple JD. Endurance exercise training during haemodialysis 
improves strength, power, fatigability and physical

performance in maintenance hemodialysis

patients. Nephrol Dial Transplant. 2005; 20(7):

1429-37. http://dx.doi.org/10.1093/ndt/gfh784 